\title{
Dual-Frequency Addressed Infrared Liquid Crystal Phase Modulators with Submillisecond Response Time
}

\author{
Xiangyi Nie \\ Thomas X. Wu
}

School of Electrical Engineering and Computer Science, University of Central Florida, Orlando, Florida

\author{
Yan-Qing Lu \\ Yung-Hsun Wu \\ Xiao Liang \\ S. T. Wu \\ College of Optics and Photonics, University of Central Florida, \\ Orlando, Florida
}

\begin{abstract}
Dynamic response of a dual-frequency liquid crystal (DFLC) based near infrared phase modulator is investigated through numerical simulations and experiments. The relationships between the optical response times and various voltage driving conditions are studied. An optical phase retarder operating at $1.55 \mu \mathrm{m}$ wavelength is demonstrated by using our homemade high figure-of-merit DFLC mixture. To improve the response time, both dual-frequency addressing and high voltage overdrive methods are employed. The obtained optical rising and decay times are reduced to less than $0.5 \mathrm{~ms}$ for a $2 \pi$ phase change. The optical transition curves simulated with finite element method show good agreement with our experimental results.
\end{abstract}

Keywords: dual frequency addressing; fast response time; finite element method; liquid crystal devices

\section{INTRODUCTION}

Phase retardation effect of liquid crystal (LC) materials supply the engine of various display applications, which are mostly in the visible wavelength range. The infrared applications of $\mathrm{LC}$ phase retarders are

This work was supported by AFOSR under contact No. F49620-01-1-0377.

Address correspondence to S. T. Wu, College of Optics and Photonics, University of Central Florida, Orlando, Florida 32816. E-mail: swu@mail.ucf.edu 
now attracting more and more research interests because of its promising commercial and military usages. Up to date, many related infrared LC devices have been demonstrated, such as the optical phased arrays, light switches, spatial light modulators, and variable optical attenuators [1-3]. As a key component of these applications, the LC phase retarder dominates the performances of a whole system. The fast dynamic response and sufficient phase retardation in various working conditions are both very critical. Although a thicker LC cell may supply more phase retardation to fulfill the need for a longer wavelength, it severely lengthens the optical response time. There is a continuous need to explore effective methods to reduce the LC response times down to the sub-millisecond level.

The most fundamental approach to improve the LC response is to develop new LC mixtures with high birefringence, large dielectric anisotropy and low viscosity [4]. Other technical efforts include elevating the operating temperature [5], inducing the polymer network [6], and the multiple-pass method [7]. However, these techniques normally complicate the fabrication process, increase the light propagation loss or have some strict technical limitations. As a consequence, the dual frequency addressing was proposed as a favorable way because it avoids many shortcomings of other alternatives [8-10].

A simple estimation may show the unique advantages of the dualfrequency addressing approach. The phase retardation of an LC cell is $\delta=2 \pi d \Delta n / \lambda$. If the employed LC material has a birefringence of $\Delta n \approx 0.2$ at an infrared wavelength $\lambda=1.55 \mu \mathrm{m}$, an LC cell with over $7 \mu \mathrm{m}$ thick cell gap must be used for a $2 \pi$ phase retarder. The director's free relaxation time is typically around $100 \mathrm{~ms}$ if the LC's elastic constants and viscosity are equivalent to those of Merck E7 mixture. Such a slow response time is obviously unacceptable. However, for dual-frequency liquid crystal (DFLC), the direction of the torque that reorients the LC alignment is varied with the addressing frequency. In the low-frequency regime, the dielectric anisotropy is positive. The torque works to align the molecules parallel to the electric field. On the contrary, the dielectric anisotropy changes sign at a high frequency, the torque then works to align the LC directors perpendicular to the field. By using this dual frequency addressing, the LC alignment becomes consistently field-controllable. Fast responses thus always can be obtained for both rise and decay processes if the driving voltage is high enough. However, the high voltage signals may only last for a short time until the cell transits to a designated phase state with a specific operating voltage. This overdriving approach has been used for normal LC cells with single frequency addressing [11]. However, in a DFLC cell, it applies for both the low and high frequencies, which 
makes the overdriving process complicated and hard to control. Furthermore, the dielectric anisotropy of a DFLC is flat in neither low frequency nor high frequency regimes. It also changes with the operating temperatures. Suitable design of the dual frequency overdriving signals thus is difficult and critical for DFLC based devices.

In this paper, we propose to use the finite element method (FEM) to simulate the DFLC's dynamic responses and optimize the detailed overdriving parameters, such as the addressing frequency, overdriving voltage, and pulse duration. A $2 \pi$ phase retarder working at $\lambda=1.55 \mu \mathrm{m}$ is thus developed using our homemade DFLC mixture. Both optical rise and decay times are shortened to less than $0.5 \mathrm{~ms}$ at room temperature, which coincide well with our FEM simulations.

\section{THEORETICAL ANALYSIS}

In a homogeneously aligned nematic LC cell, the electric field is almost perpendicular to the initial LC directors. To minimize the electric field energy density, the direction of LC's largest dielectric constant tends to orient along the external field, which normally induces a director tilting. However, the electric field should be large enough to resist the elastic torque. A threshold voltage thus could be deduced from the static Euler-Lagrange equation as $V_{t h}=\pi \sqrt{K_{11} / \Delta \varepsilon}$, assuming a small angle molecular deformation and using the single elastic constant approximation $\left(K_{11}=K_{22}=K_{33}\right)$. As the applied voltage exceeds $V_{\text {th }}$, the director starts to tilt. To guide the LC directors relaxation direction, the cell has a small pretilt angle $\theta_{p}$, which is the angle between the LC director and the substrate surface. A higher voltage results in a larger $\theta$ angle and faster director deformation. This dynamic process is described with the Erickson-Leslie equation [12,13]

$$
K_{11} \frac{\partial^{2} \theta}{\partial z^{2}}+\varepsilon_{o} \Delta \varepsilon E^{2} \theta=\gamma_{1} \frac{\partial \theta}{\partial t}
$$

where the backflow and inertial effects are ignored to simplify the equation. $\gamma_{1}$ is the rotational viscosity, $K_{11}$ represents the splay elastic constants, and $\Delta \varepsilon$ is the dielectric anisotropy. In the rising process, the applied electric field reorients LC directors. Adopting the small angle approximation, the rise time between two phase states (e.g., $10 \%$ and $90 \%$ phase retardations) is derived to be proportional to

$$
\tau_{\text {rise }}=\frac{\tau_{0}}{\left(V / V_{t h}\right)^{2}-1}
$$

where $\tau_{0}=\gamma_{1} d^{2} / K_{11} \pi^{2}$. As Eq. (2) shows, the rise time may be improved by simply elevating the voltage amplitude. 
However, for both positive $(\Delta \varepsilon>0)$ and negative $(\Delta \varepsilon<0)$ LC materials, the external electric field can only orient LC directors in the rise process. LC directors' decay is only dependent on the LC material's physical properties. The decay is an exponential process with time constant $\tau_{o}$, which can not be speeded up by external electric field. Long decay time can significantly deteriorate LC device performance, especially for a thick LC cell employed in the infrared spectral region.

The dual-frequency addressing is an effective method that works specifically to shorten the LC's decay time. In a DFLC mixture, assuming the low and high driving frequencies are $f_{\ell}$ and $f_{h}$. The corresponding dielectric anisotropies are $\Delta \varepsilon_{l}$ and $\Delta \varepsilon_{h}$, respectively. Because of the negative $\Delta \varepsilon_{h}$, a high frequency electrical field thus can be applied to accelerate the director relaxation process. In this case, the decay time equation is modified to be

$$
\tau_{\text {decay }}=\frac{\tau_{o}}{\left|\frac{\Delta \varepsilon_{h}}{\Delta \varepsilon_{\ell}}\right|\left(\frac{V}{V_{t h}}\right)^{2}+1}
$$

which is similar to the expression of $\tau_{\text {rise }}$. As a consequence, the overdriving technique that is originally valid for only the rising process now also can be extended to the decay process. Some ultrafast (e.g., sub-millisecond) nematic LC devices may be developed, given the availability of suitable DFLC materials.

To further study a DFLC device dynamic behavior and design detailed overdriving schemes, a numerical simulation is desired because it reveals the internal processes of $\mathrm{LC}$ reorientation with higher accuracy.

\section{NUMERICAL SIMULATION}

The finite element method (FEM) is a well recognized numerical simulation method. A general purpose FEM computer program can be easily developed to rigorously analyze the problems where analytical solutions are difficult to obtain. Dual frequency and overdriving effects need to be considered in the analysis, which are beyond the limitation of most commercially available LC simulation software. Thus numerical simulation based on FEM is developed as an effective design tool of our proposed DFLC devices.

The numerical simulation of a DFLC device's dynamic behavior is based on the assumption that the system acts to minimize its free energy. The dynamic evolution of the LC director $n_{i}$ are provided by 
the analysis of the electric and elastic properties of the DFLC material, which is given by the Oseen-Frank free-energy equation,

$$
F=\int_{v}\left[\frac{1}{2} K_{11}(\nabla \cdot \widehat{n})^{2}+\frac{1}{2} K_{22}(\widehat{n} \cdot \nabla \times \widehat{n})^{2}+\frac{1}{2} K_{33}(\widehat{n} \times \nabla \times \widehat{n})^{2}-\frac{1}{2} D \cdot E\right] d v,
$$

where $K_{11}, K_{22}$, and $K_{33}$ are the three Frank elastic constants, the electric energy part of the free energy $(1 / 2) D \cdot E$ can be written as $(1 / 2) \varepsilon_{0} \overleftrightarrow{\varepsilon} E \cdot E=(1 / 2) \varepsilon_{0} \overleftrightarrow{\varepsilon} \nabla V \cdot \nabla V$. For DFLC, frequency dependent dielectric tensor $\stackrel{\leftrightarrow}{\varepsilon}$ has the expression of $\varepsilon_{l m}=\varepsilon_{\perp}(f) \delta_{l m}+\Delta \varepsilon(f) \cdot n_{l} n_{m}$, where $\mathrm{l}, \mathrm{m}=\{\mathrm{x}, \mathrm{y}, \mathrm{z}\}$. The dielectric anisotropy of a DFLC is defined as $\Delta \varepsilon(f)=\varepsilon_{\|}(f)-\varepsilon_{\perp}(f)$, where $\varepsilon_{\|}(f)$ and $\varepsilon_{\perp}(f)$ are the frequency-dependent permittivities parallel and perpendicular to the LC directors. The $\varepsilon_{\perp}(f)$ of a DFLC is usually much inert to the driving frequency than $\varepsilon_{\|}(f)$, so $\varepsilon_{\perp}(f)$ can be assumed as a constant, and $\varepsilon_{\|}(f)$ is taken as a variable. To describe this effect in the simulation program, we measure the frequency dependent dielectric properties of our homemade DFLC and implement them in the computer program. Since response time is highly dependent on $\Delta \varepsilon(f)$, the driving frequencies need to be optimized in the simulation to achieve the shortest response time. For a homogeneous aligned DFLC phase retarder, Eq. (4) can be simplified as

$$
F=\frac{1}{2} \int_{0}^{d}\left\{\left(K_{11} \cos ^{2} \theta+K_{33} \sin ^{2} \theta\right)\left(\frac{d \theta}{d z}\right)^{2}-\frac{D_{z}}{\varepsilon_{0}\left[\varepsilon_{\perp}+\Delta \varepsilon(f) \sin ^{2} \theta\right]}\right\} d z .
$$

Equation (5) describes the free energy of our DFLC phase retarder.

In the FEM program, vector representation of LC directors is used to avoid the instability of $\theta-\phi$ representation in the high voltage regime. We derive the dynamic equation for LC directors by considering the rotational viscosity $\gamma_{1}$ and ignoring the flow effects,

$$
n_{i}^{\text {new }}=n_{i}^{\text {old }}-\frac{\Delta t}{\gamma_{1}}[F]_{n_{i}}
$$

In each evolution step, $\mathrm{n}_{\mathrm{i}}$ needs to be normalized as a unit. Strong anchoring is considered, and the boundary condition in the FEM simulation is described by prescribing some pretilt angles on the DFLC surface planes. Once the DFLC directors are obtained, optical transmittance is calculated by the extended Jones matrix approach $[14,15]$. 
Most commercially available DFLC mixtures have a low birefringence, high viscosity, and small $|\Delta \varepsilon|$ values. To overcome these drawbacks, we prepared a high birefringence and low viscosity DFLC mixture using $30 \%$ biphenyl esters and $70 \%$ lateral difluoro tolanes. The physical properties of our DFLC mixture at room temperature $\left(\mathrm{T}=22^{\circ} \mathrm{C}\right.$ ) are listed as follows: crossover frequency $f_{c} \approx 4 \mathrm{kHz}$ and $\Delta n=n_{e}-n_{o}=0.25$ at $\lambda=1.55 \mu \mathrm{m}$. Beside the response times, several other reasons are also considered for best driving frequencies, such as $\Delta \varepsilon$ tolerance of environment temperature, and heating effect on the LC

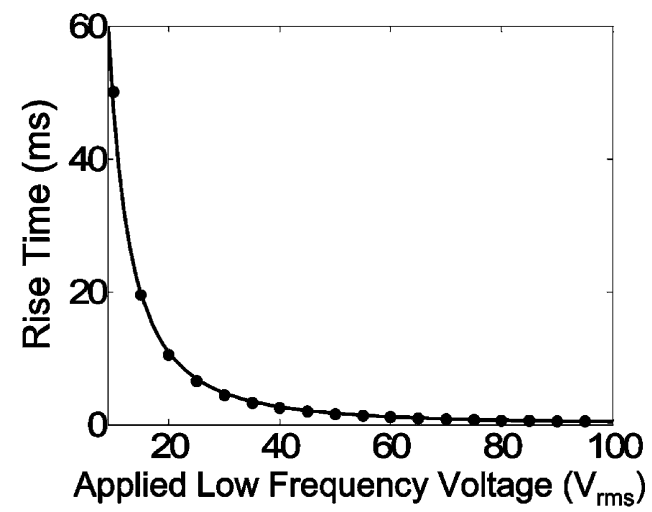

(a)

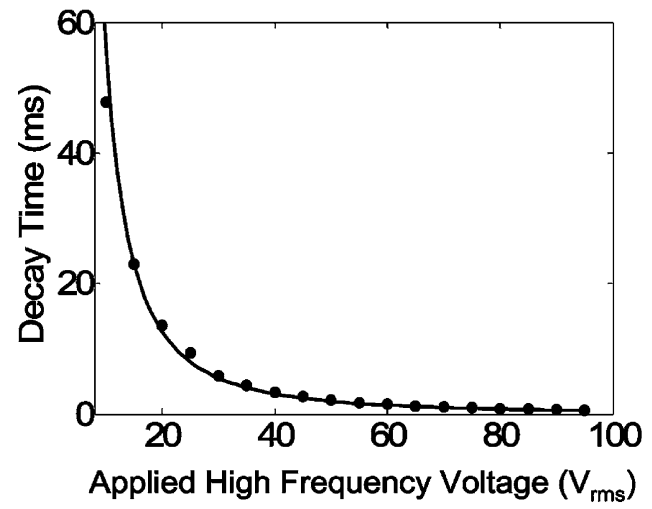

(b)

FIGURE 1 (a) Rise time of $2 \pi$ phase change as a function of applied low frequency $\left(f<f_{\mathrm{c}}\right)$ voltage; (b) decay time of $2 \pi$ phase change dependent on the applied high frequency $\left(f>f_{\mathrm{c}}\right)$ voltage. DFLC cell gap $\mathrm{d}=9.2 \mu \mathrm{m}$ and $\lambda=1.55 \mu \mathrm{m}$. 
cell. Through our simulation and consideration of the abovementioned factors, $1 \mathrm{kHz}$ low frequency and $30 \mathrm{kHz}$ high frequency are chosen, where $\Delta \varepsilon=4.7$ at $f=1 \mathrm{kHz}$ and $\Delta \varepsilon=-4.0$ at $f=30 \mathrm{kHz}$.

Overdriving effect is also considered and the driving voltages are optimized through the simulation. As a result, a 9.2- $\mu \mathrm{m}$-thick homogenous alignment cell is used and overdriving pulse voltages for both rise and decay processes are determined for achieving sub-millisecond response time. Figure $1(\mathrm{a})$ is the calculated rise time for achieving $2 \pi$ phase change under different overdrive voltages. The simulation result shows that $0.5 \mathrm{~ms}$ rise time can be realized if a $93 \mathrm{~V}_{\text {rms }}$ overdrive voltage is applied, which is very close to our experimental result of $95 \mathrm{~V}_{\text {rms }}$. Figure $1(\mathrm{~b})$ plots the decay time as a function of the applied high frequency voltage. To obtain $0.5 \mathrm{~ms}$ decay time, the required erasing voltage is $\sim 79 \mathrm{~V}_{\text {rms }}$.

In our DFLC phase retarder system, a $2.5 \mathrm{~V}_{\text {rms }}$ bias voltage of $1 \mathrm{kHz}$, which is slightly lower than the threshold voltage, is used to reduce the delay time during the rise period. An initial DFLC director distribution corresponding to the specific bias voltage was used to simulate its effect. The simulation shows that without the bias voltage the rise time would be $0.7 \mathrm{~ms}$ longer under the same overdriving condition.

\section{EXPERIMENTS}

Figure 2 plots the experimental setup. A $9.2 \mu \mathrm{m}$ DFLC cell was placed between two crossed sheet polarizers. The experiments were carried out at room temperature $22^{\circ} \mathrm{C}$. As mentioned above, the low addressing

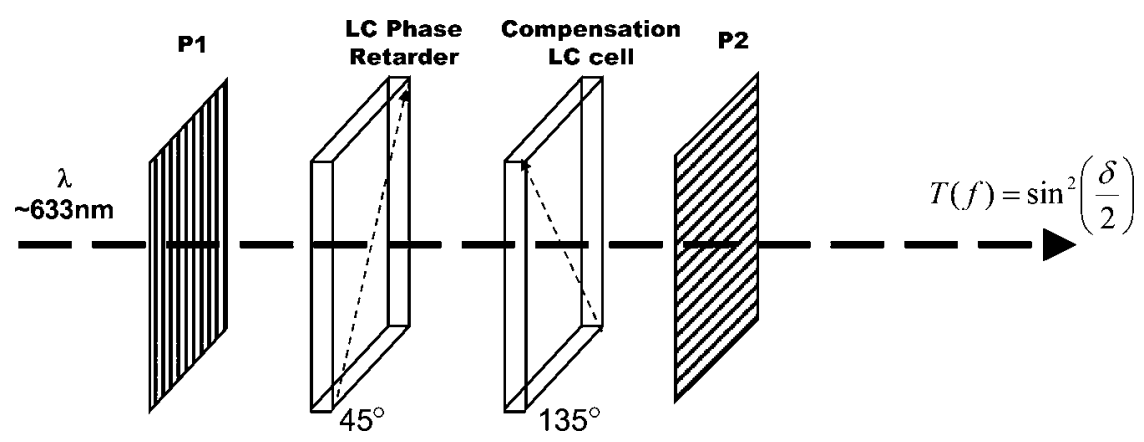

FIGURE 2 Experimental setup. A DFLC phase retarder is placed between a pair of crossed polarizers. A compensation LC cell is used for convenient detection. The DFLC cell is driven under overdrive and frequency modulation conditions. 
frequency is $1 \mathrm{kHz}$ and the high one is $30 \mathrm{kHz}$. To demonstrate an infrared phase retarder, we used an Ando AQ4321D tunable laser operating at $\lambda=1.55 \mu \mathrm{m}$ as a light source. The output transmittance

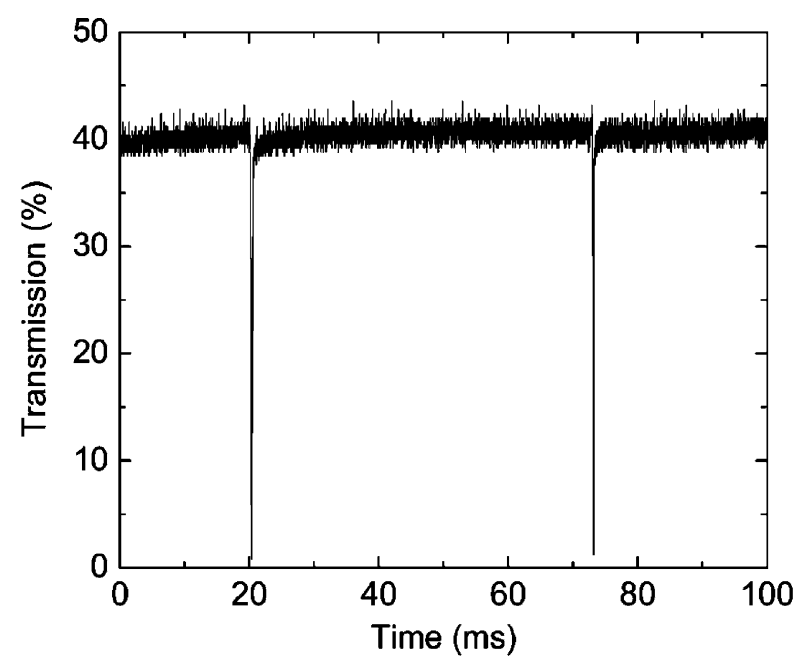

(a)

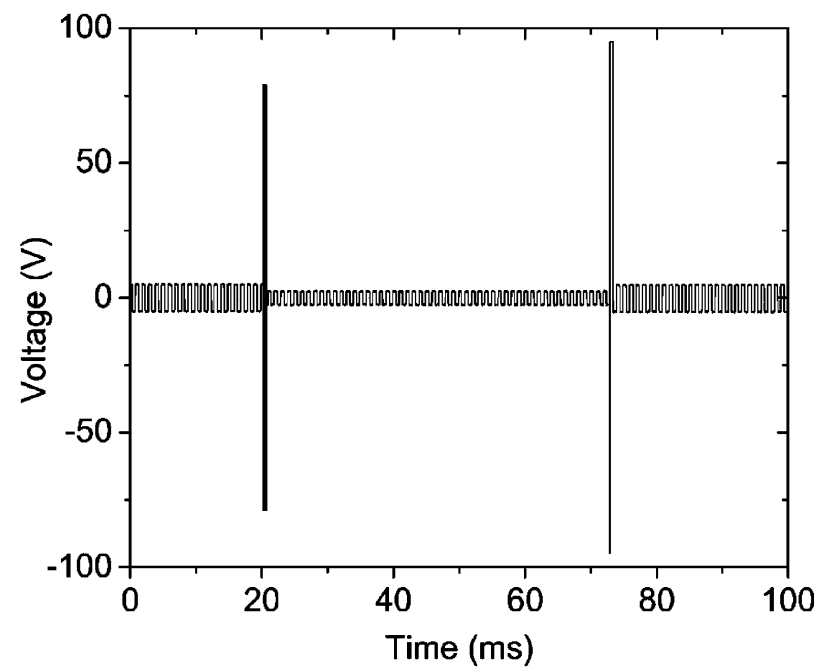

(b)

FIGURE 3 (a) Decay and Rise processes, (b) Corresponding applied voltage. Ultra-fast response time of $2 \pi$ phase retardation at $\lambda=1.55 \mu \mathrm{m}$ is achieved by high voltage overdriving pulses and frequency modulation. 
was measured using an InGaAs photodiode detector. A computer controlled LabVIEW system was used for data processing. The transmittance of such a phase retarder is $\mathrm{I}=\mathrm{I}_{0} \sin ^{2}[\delta / 2]$, where $\mathrm{I}_{0}$

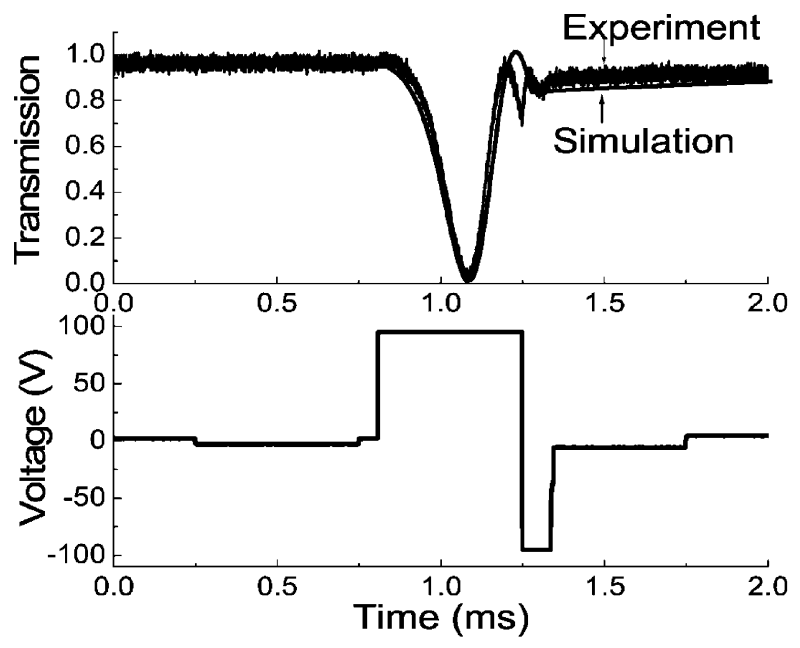

(a)
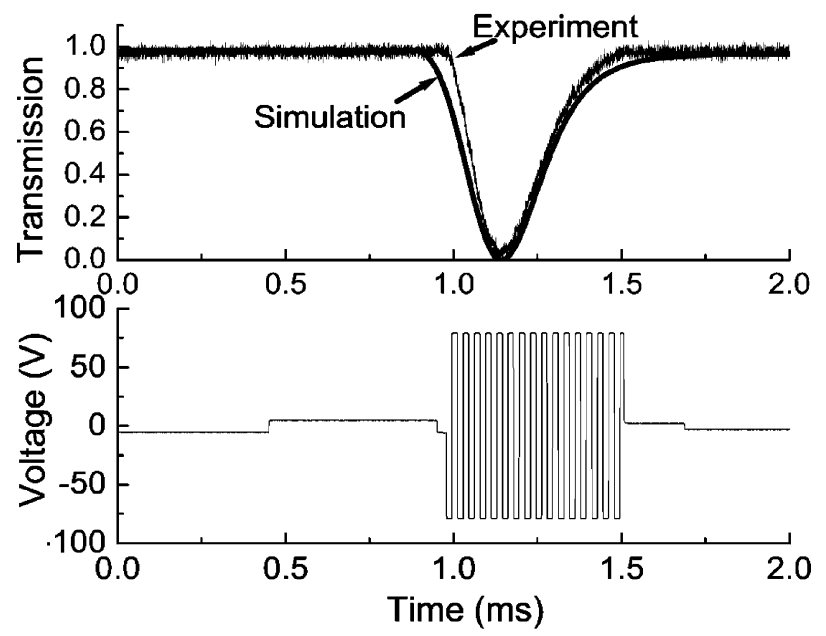

(b)

FIGURE 4 (a) Rising state (b) decay state. Upper part of each figure shows the comparison between the experimental and simulation results, the lower part shows the corresponding voltage and frequency modulation. Numerical simulation shows the dual-frequency overdrive and bias voltage effects. 
is the incident light intensity and $\delta$ is the LC phase retardation. The LC director reorientation can be revealed from the light transmission and our simulation results compared with experiment.

Figure 3 exhibits the measured optical response of our DFLC phase retarder, where Figure 3(a) shows the light transmittance as a function of time and the driving signals are displayed in Figure 3(b). In the experiments, $1 \mathrm{kHz} 2.5 \mathrm{~V}_{\mathrm{rms}}$ bias voltage and $95 \mathrm{~V}_{\mathrm{rms}}$ overdriving pulses, as proposed from our numerical simulation, were applied to the cell to reorient the LC directors. The overdriving voltage only lasts about $0.5 \mathrm{~ms}$ to avoid over-tilting. Then a $5 \mathrm{~V}_{\text {rms }}$ low frequency $(1 \mathrm{kHz})$ voltage is applied to hold the LC directors. The cell was kept at a $2 \pi$ phase retardation status before the high frequency voltage was applied. The detailed rising process is illustrated in Figure 4(a) and compared with the simulation results. The experimental curve coincides well with the theoretical prediction. Both of them show a $2 \pi$ phase transition within less than $0.5 \mathrm{~ms}$ for the rise process.

For the decay process, the LC directors are already tilted up, which is so called homeotropic state. A high frequency $(30 \mathrm{kHz}), 79 \mathrm{~V}_{\mathrm{rms}}$ voltage was used to drive the LC cell back to its original homogeneous distribution. From Figure 4(b), $2 \pi$ phase decay is achieved within only $\sim 0.5 \mathrm{~ms}$, which also agrees well with our theoretical estimation.

From our experiments, both rise and decay times of a $2 \pi$ phase retarder at $\lambda=1.55 \mu \mathrm{m}$ are shortened to $\sim 0.5 \mathrm{~ms}$ because of the dual frequency overdriving effect. The repetition rate can reach $1 \mathrm{kHz}$, which is quite impressive for a LC device. For comparison, we also measured the response times of an identical cell filled with the same DFLC driven at $5 \mathrm{~V}_{\text {rms }}$. The rise and decay times were measured to be $500 \mathrm{~ms}$ and $350 \mathrm{~ms}$, respectively, which are about two orders of magnitude slower. The dual-frequency overdriving technique is indeed a simple and effective way to accelerate the LC responses.

\section{CONCLUSION}

We demonstrated a $2 \pi$ phase retarder working at $\lambda=1.55 \mu \mathrm{m}$ using our DFLC material. Through voltage and frequency modulations, a relatively thick LC cell $(9.2 \mu \mathrm{m})$ can be switched within sub-millisecond $(<0.5 \mathrm{~ms})$ for both rise and decay processes. Frequency dependent dielectric anisotropy and overdriving schemes are considered in finite element simulation. The numerical simulation serves as an effective analysis and design tool of the DFLC device, and its results agree well with the experimental demonstrations. 


\section{REFERENCES}

[1] McManamon, P. F., Dorschner, T. A., Corkum, D. L., Friedman, L., Hobbs, D. S., Holz, M., Liberman, S., Nguyen, H. Q., Resler, D. P., Sharp, R. C., \& Watson, E. A. (1996). Proc. IEEE, 84, 268.

[2] Mao, C., Xu, M., Feng, W., Huang, T., Wu, K., \& Wu, J. (2003). Proc. SPIE, 5003, 121.

[3] Lu, Y. Q., Wong, C., \& Wu, S. T. (2004). IEEE Photon. Tech. Lett., 16, 861.

[4] Gauza, S., Wang, H., Wen, C. H., Wu, S. T., Seed, A., \& Dabrowski, R. (2003). J. Appl. Phys. Part 1, 42, 3463.

[5] Wu, S. T. \& Wu, C. S. (1989). J. Appl. Phys., 65, 527.

[6] Fan, Y. H., Lin, Y. H., Ren, H., Gauza, S., \& Wu, S. T. (2004). Appl. Phys. Lett., $84,1233$.

[7] Wu, S. T. (1990). Appl. Phys. Lett., 57, 986.

[8] Bucher, H. K., Klingbiel, R. T., \& VanMeter, J. P. (1974). Appl. Phys. Lett., 25, 186.

[9] Khoo, I. C. \& Wu, S. T. (1993). Optics and Nonlinear Optics of Liquid Crystals, World Scientific: Singapore.

[10] Golovin, A. B., Shiyanovskii, S. V., \& Lavrentovich, O. D. (2003). Appl. Phys. Lett., 83, 3864.

[11] Wu, S. T., Efron, U., \& Hess, L. D. (1984). Appl. Opt., 23, 3911.

[12] Erickson, J. L. (1961). Trans. Soc. Rheol., 5, 23.

[13] Leslie, F. M. (1968). Arch. Ration. Mechan. Anal., 28, 265.

[14] Jones, R. C. (1942). J. Opt. Soc. Am., 32, 486.

[15] Lien, A. (1997). Liq. Cryst., 22, 171. 